\title{
УДК 621.831.1:539.4 \\ ПРОЕКТИРОВАНИЕ ТЯЖЕЛО НАГРУЖЕННОЙ ЦИКЛОИДАЛЬНОЙ ПЕРЕДАЧИ СО СВОБОДНОЙ ОБОЙМОЙ ИЗ УСЛОВИЯ КОНТАКТНОЙ ПРОЧНОСТИ
}

\author{
Ефременков Егор Алексеевич1,2, \\ ephrea@mail.ru
}

\author{
Ефременкова Светлана Константиновна ${ }^{1}$, \\ efremenkova@tpu.ru \\ 1 Национальный исследовательский Томский политехнический университет, \\ Россия, 634050, г. Томск, пр. Ленина, 30. \\ 2 Томский государственный университет систем управления и радиоэлектроники, \\ Россия, 634050, г. Томск, пр. Ленина, 40.
}

\begin{abstract}
Актуальность исследования обусловлена потребностью в проектировании тяжелонагруженных механизмов для горнодобывающей промышленности, например проходческих комбайнов, транспортеров и др., а также отсутствием методик для предварительного определения геометрических параметров таких механизмов на основе циклоидальной передачи с промежуточными телами качения и свободной обоймой из условия контактной прочности. Разработка такой методики позволяет применять в приводах проходческих и транспортных механизмов компактные и энергосберегающие передачи с обоснованной надежностью и долговечностью.

Цель: определение зависимости одного из основных геометрических параметров передачи с промежуточными телами качения и свободной обоймой из условия контактной прочности, а также разработка графических материалов для выбора кинематического коэффрициента передачи, входящего в итоговую зависимость.

объекты: ииклоидальные профиля колес передачи в местах контакта с промежуточными телами качения, а также основные геометрические параметры колес необходимые для их проектирования.

Методы: аналитические методы теории зубчатых зацеплений и напряженно-деформационного состояния детали.

Результаты. Представлены основные формулы для определения геометрического параметра передачи с промежуточными телами качения и свободной обоймой из условия контактной прочности, получены выражение для определения минимально допустимого значения геометрического параметра передачи с промежуточными телами качения и свободной обоймой, представлена гистограмма определения кинематического коэфффициента передачи с промежуточными телами качения и свободной обоймой по заданному коэфффициенту смещения и числу тел качения. Полученные результаты будут способствовать проектированию надежных компактных энергоэфффективных механизмов для тяжельх условий работы, что особенно востребовано в горнодобывающей и нефтегазовой промьшленности.
\end{abstract}

\section{Ключевые слова:}

Тяжелонагруженный механизм, горнодобывающее оборудование, циклоидальная передача, промежуточные тела качения, условие контактной прочности, циклоидальный профриль, кинематический коэфффициент, свободная обойма.

\section{Введение}

В современных машинах и механизмах все более востребованы передачи с промежуточными телами качения (ПТК). Они уже применяются в нефтегазовой отрасли, в механизмах теплоэлектростанций, транспортных системах [1], горнодобывающей промышленности [2]. В частности, передачи с ПТК используются в приводах перемешивателей бурового раствора, горно-шахтных комбайнов и других тяжелонагруженных механизмах, которые работают длительное время без обслуживания.

К высоконагруженным машинам предъявляются высокие требования по надежности, энергоэффективности, уравновешенности [3, 4]. Для высоконагруженных машин, таких как горнопроходческие и горнодобывающие, наиболее важным параметром является высокая нагрузочная способность передач, входящих в их состав, и способность воспринимать значительные перегрузки, сохраняя при этом работоспособность. Обеспечить горной машине такие характеристики позволяет передача с промежуточными телами качения и свободной обоймой (ПТКСО).
Передача с ПТКСО (рис. 1) обладает комплексом высоких технических характеристик: компактностью, высокой точностью зацепления, способностью передавать большие крутящие моменты, неприхотливостью в обслуживании, пониженным трением скольжения в зацеплении и, как следствие, высоким КПД. Обеспечить передаче высокую перегрузочную способность позволяет многопарность зацепления.

При проектировании механических передач, в том числе и для горных машин, определяющим показателем прочности является площадь поперечного сечения несущих деталей передачи. А размеры сечения определяются исходя из условия контактной прочности и влияют на размеры самой передачи и наоборот. В передаче с ПТКСО определяющим нагрузочную способность показателем является контактное напряжение несущих поверхностей взаимодействующих деталей. Проектируя тяжелонагруженный механизм, важно понимать на начальном этапе его ориентировочные размеры с целью соблюдения требуемого габарита и корректировки исходных расчетных параметров. Поэтому определение геометрической характеристики передачи с ПТКСО из условия контактной 
прочности является актуальным для проектирования высоконагруженных механизмов для горных работ при проведении инженерных расчетов.

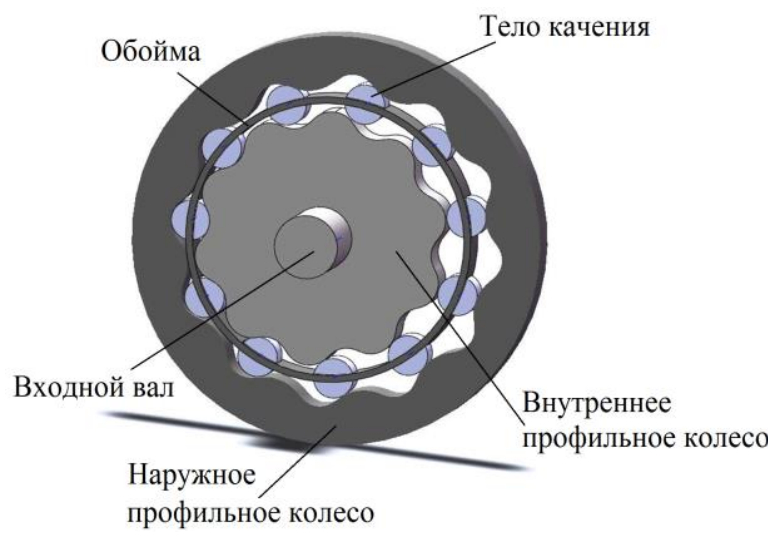

Рис. 1. Схема передачи с промежуточными телами качения и свободной обоймой

Fig. 1. Scheme of transmission with intermediate rolling bodies and free cage

\section{Анализ контактных напряжений в зацеплении передачи и определение радиуса центров из условия контактной прочности}

Передачи с промежуточными телами качения изучаются достаточно давно [1, 5-7], но до сих пор их применение ограничено. Одной из причин является отсутствие обоснования выбора размеров передачи исходя из условия контактной прочности. Исследованием передач с ПТК, циклоидального зацепления и расчетом их силовых характеристик вообще и контактных напряжений в частности занимаются ученые в России, Белоруссии, Китае и других странах [5-17], но выражения для определения геометрических параметров передачи с ПТКСО, исходя из условия контактной прочности, представлено не было. Поэтому целью работы является получение выражения для определения геометрической характеристики передачи с ПТКСО, такой как радиус положения центров тел качения, через исходные параметры. Получив на ранних стадиях проектирования значение радиуса положения центров тел качения, уже можно наглядно представить примерные габаритные размеры всего механизма. В то время как значение любого из исходных параметров передачи не дает такого представления. Исходными параметрами для расчета передачи с ПТКСО являются [5]: $r_{2}$ - радиус производящей окружности, $Z_{2}$ - число тел качения, $\chi$ - коэффициент смещения и $r_{\mathrm{b}}$ - радиус тела качения.

Условие контактной прочности записывается как [18]:

$$
\left(\sigma_{\mathrm{H}}\right)_{\mathrm{p}} \leq\left[\sigma_{\mathrm{H}}\right]
$$

где $\left(\sigma_{H}\right)_{p}$ - расчетное контактное напряжение; $\left[\sigma_{H}\right]-$ допустимое напряжение на контактную прочность.

Из формулы Герца $[19,20]$ для двух контактирующих цилиндров можно получить формулу для определения контактных напряжений на $i$-м теле качения передачи с ПТКСО [5]:

$$
\left(\sigma_{\mathrm{H}}\right)_{i}=\sqrt{\frac{F_{i}\left(\rho_{2 i} \pm \rho_{1 i}\right)}{\pi l_{b} \rho_{1 i} \rho_{2 i}\left(\frac{1-\mu_{1}^{2}}{E_{1}}+\frac{1-\mu_{2}^{2}}{E_{2}}\right)}},
$$

где $F_{i}$ - нормальная сила к поверхности контакта колеса с $i$-м телом качения; $\rho_{1 i}, \rho_{2 i}$ - радиусы кривизны в точке контакта контактирующих тел - профиля колеса и тела качения соответственно; $\mu_{1}, \mu_{2}$ - коэффициенты Пуассона для материалов колеса и тела качения соответственно; $E_{1}, E_{2}-$ модули упругости для первого и второго контактирующих тел; $l_{b}$ - длина тела качения.

Знак «+» используется для выпукло-выпуклого контакта, а знак «-» - для выпукло-вогнутого.

Для определения нормальной силы рассмотрим схему распределения усилий в зацеплении передачи с ПТКСО (рис. 2).

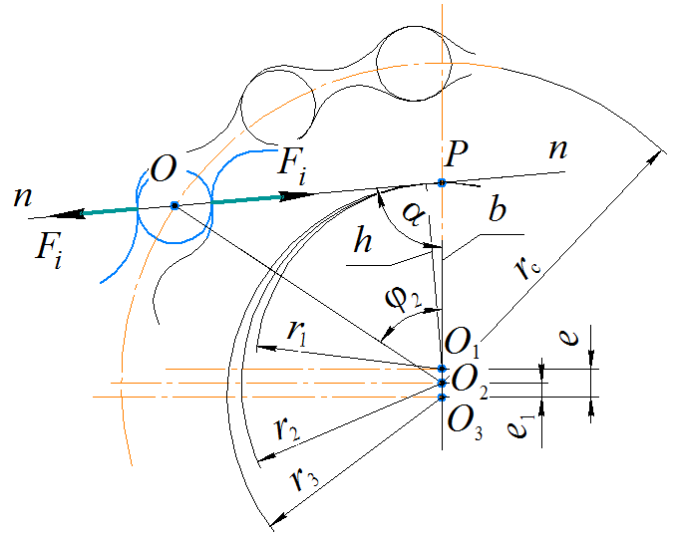

Pис. 2. Расчетная схема определения усилий в зачеплении передачи с промежуточными телами качения и свободной обоймой

Fig. 2. Calculation scheme for determining forces in engagement of transmission with intermediate rolling bodies and free cage

На рис. 2 обозначено: $P$ - полюс зацепления; $O_{1}$, $\mathrm{O}_{2}, \mathrm{O}_{3}$ - центры кулачка, обоймы с телами качения и венца соответственно; $r_{1}, r_{2}, r_{3}$ - радиусы центроид кулачка, обоймы и венца соответственно; $r_{\mathrm{c}}$ - радиус центров тел качения; $\varphi_{2}$ - угол поворота обоймы с телами качения; $e_{1}$ - эксцентриситет зацепления; $e-$ полый эксцентриситет передачи; $F_{i}$ - усилие в зацепление венца/кулачка и $i$-го тела качения; $h$ - кратчайшее расстояние от центра венца/кулачка до линии действия $i$-го усилия в зацеплении.

Крутящий момент на внутреннем циклоидальном колесе передачи с ПТКСО определяется как

$$
T_{\mathrm{K}}=\sum F_{i} h_{i} .
$$

Известно [21], что максимальная сила $F_{\max }$ будет, когда угол $\alpha=90^{\circ}$ (рис. 2), тогда

$$
F_{\text {max }}=\frac{T_{\mathrm{K}} b}{\sum h_{i}^{2}}
$$

и

$$
\frac{F_{i}}{h_{i}}=\frac{F_{\max }}{b} \rightarrow F_{i}=\frac{F_{\max } h_{i}}{b} .
$$


Так, выражение через крутящий момент нормальной силы в контакте с $i$-м телом качения запишем в виде

$$
F_{i}=\frac{T_{\mathrm{K}} b}{\sum h_{i}^{2}} \frac{h_{i}}{b}=\frac{T_{\mathrm{\kappa}} h_{i}}{\sum h_{i}^{2}} .
$$

Если принять, что колеса с циклоидальным профилем и тела качения изготавливают из одного материала, как это в основном бывает, то условие контактной прочности с учетом выражений (1) и (2) запишем в виде:

$$
\left(\sigma_{\mathrm{H}}\right)_{\max }=\sqrt{\frac{T_{\mathrm{\kappa}} h_{i} E\left(\rho_{2 i} \pm \rho_{1 i}\right)}{2 \pi l_{b} \rho_{1 i} \rho_{2 i}\left(1-\mu^{2}\right) \sum h_{i}^{2}}} \leq\left[\sigma_{\mathrm{H}}\right] .
$$

Радиусы кривизны через исходные параметры передачи с ПТКСО определены в работе [22]. Так, радиус кривизны циклоидального профиля внутреннего колеса передачи выражается как

$$
\begin{gathered}
\rho_{1}=r_{2} \sqrt{1+\chi^{2}-2 \chi \cos \varphi}-r_{b}- \\
-\frac{Z_{2} r_{2} i_{21} \sqrt{1+\chi^{2}-2 \chi \cos \varphi}}{Z_{1} \cos \varphi+\frac{\chi^{2} \sin ^{2} \varphi}{(1-\chi \cos \varphi)}+Z_{2}(1-\chi \cos \varphi)},
\end{gathered}
$$

где $i_{21}$ - передаточное отношение от тел качения к внутреннему колесу передачи, и определяется из выражения:

$$
i_{21}=1-\frac{1}{Z_{2}} .
$$

Для краткости записи обозначим

$$
a=\sqrt{1+\chi^{2}-2 \cos \varphi} .
$$

Тогда выражение для суммы и произведения радиусов кривизны внутреннего циклоидального колеса передачи с ПТКСО и тела качения через исходные параметры после преобразований выражения (4) можно представить следующим образом:

$$
\begin{gathered}
\times\left(1-\frac{\rho_{2}+\rho_{1}=r_{2} a \times}{\chi Z_{1} \cos \varphi+\frac{\chi^{2} \sin ^{2} \varphi}{(1-\chi \cos \varphi)}+Z_{2}(1-\chi \cos \varphi)}\right) \\
\times\left(1-\frac{r_{b}}{r_{2} a}-\frac{\rho_{2} \rho_{1}=r_{b} r_{2} a \times}{\chi Z_{1} \cos \varphi+\frac{\chi^{2} \sin ^{2} \varphi}{(1-\chi \cos \varphi)}+Z_{2}(1-\chi \cos \varphi)}\right)
\end{gathered}
$$

Обозначим здесь

$$
k=\left(1-\frac{Z_{2} i_{21}}{\chi Z_{1} \cos \varphi+\frac{\chi^{2} \sin ^{2} \varphi}{(1-\chi \cos \varphi)}+Z_{2}(1-\chi \cos \varphi)}\right),
$$

тогда выражения (5) и (6) примут вид

$$
\rho_{2}+\rho_{1}=k r_{2} a
$$

$$
\rho_{2} \rho_{1}=r_{b} r_{2} a\left(k-\frac{r_{b}}{r_{2} a}\right)
$$

Отношение суммы радиусов кривизны к их произведению запишем в виде

$$
\frac{\rho_{2}+\rho_{1}}{\rho_{2} \rho_{1}}=\frac{k}{r_{b}\left(k-\frac{r_{b}}{r_{2} a}\right)} .
$$

Выражение расстояния $h$ (3) через исходные параметры запишется так:

$$
h_{i}=\frac{i_{21} r_{2} \chi \sin \varphi}{\sqrt{1+\chi^{2}-2 \cos \varphi}} .
$$

Тогда отношение расстояния до $i$-й нормали к сумме квадратов всех расстояний окончательно запишется следующей формулой:

$$
\frac{h_{i}}{\sum h_{i}^{2}}=\frac{\sin \varphi}{a r_{2} \chi i_{21} \sum\left(\frac{\sin \varphi}{a}\right)^{2}} .
$$

Подставив выражения (8) и (9) в (2) получим:

$$
\left(\sigma_{\mathrm{H}}\right)_{i}=\sqrt{\frac{T_{\mathrm{K}} E k \sin \varphi}{2 \pi l_{b}\left(1-\mu^{2}\right) r_{b}\left(k-\frac{r_{b}}{r_{2} a}\right) a r_{2} \chi i_{21} \sum\left(\frac{\sin \varphi}{a}\right)^{2}}} .
$$

В выражении (10) произведение $r_{2} \chi$ определяет радиус положения центров тел качения $r_{c}$, который является одним из геометрических показателей передачи с ПТКСО, определяющих ее габаритные размеры. Полагаем, что колеса передачи и тела качения изготавливаются из стали. Тогда вынесем из-под корня постоянные величины и запишем условие контактной прочности для передачи с ПТКСО как:

$$
=191,65 \cdot 10^{3} \sqrt{\frac{\left(\sigma_{\mathrm{H}}\right)_{\max }}{T_{\mathrm{K}} k \sin \varphi}} \leq\left[\sigma_{\mathrm{H}}\right] .
$$

Из условия контактной прочности (11) выразим

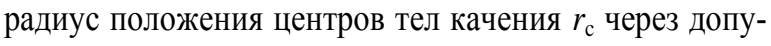
стимое контактное напряжение

$$
r_{c}>\frac{36,73 \cdot 10^{9} T_{\kappa} k \sin \varphi}{l_{b} r_{b} a i_{21}\left[\sigma_{\mathrm{H}}\right]^{2}\left(k-\frac{r_{b}}{r_{2} a}\right) \sum\left(\frac{\sin \varphi}{a}\right)^{2}} .
$$

В передаче с ПТКСО максимальное контактное напряжение возникает при расположении тел качения на угле $\varphi \approx 70^{\circ}$. Так, принимая, что в любой передаче с ПТКСО максимальное контактное напряжение будет определяться при $\varphi=70^{\circ}$, все последующие вычисления будем производить используя это значение угла $\varphi$.

Тогда используя выражение (7) определяем значение коэффициента $k$ при указанном угле $\varphi$ для различных значений $Z_{2}$ (числа тел качения) и коэффициента $\chi$. Подставляя в выражение (7) $Z_{2}$ в диапазоне от 8 до 40 с шагом 1 и значения $\chi$ из рекомендованного диапазона [5] с шагом 0,01, получим гистограмму 
(рис. 3) определения коэффициента $k$ в зависимости от изменения коэффициента смещения $\chi$ для различного числа тел качения.

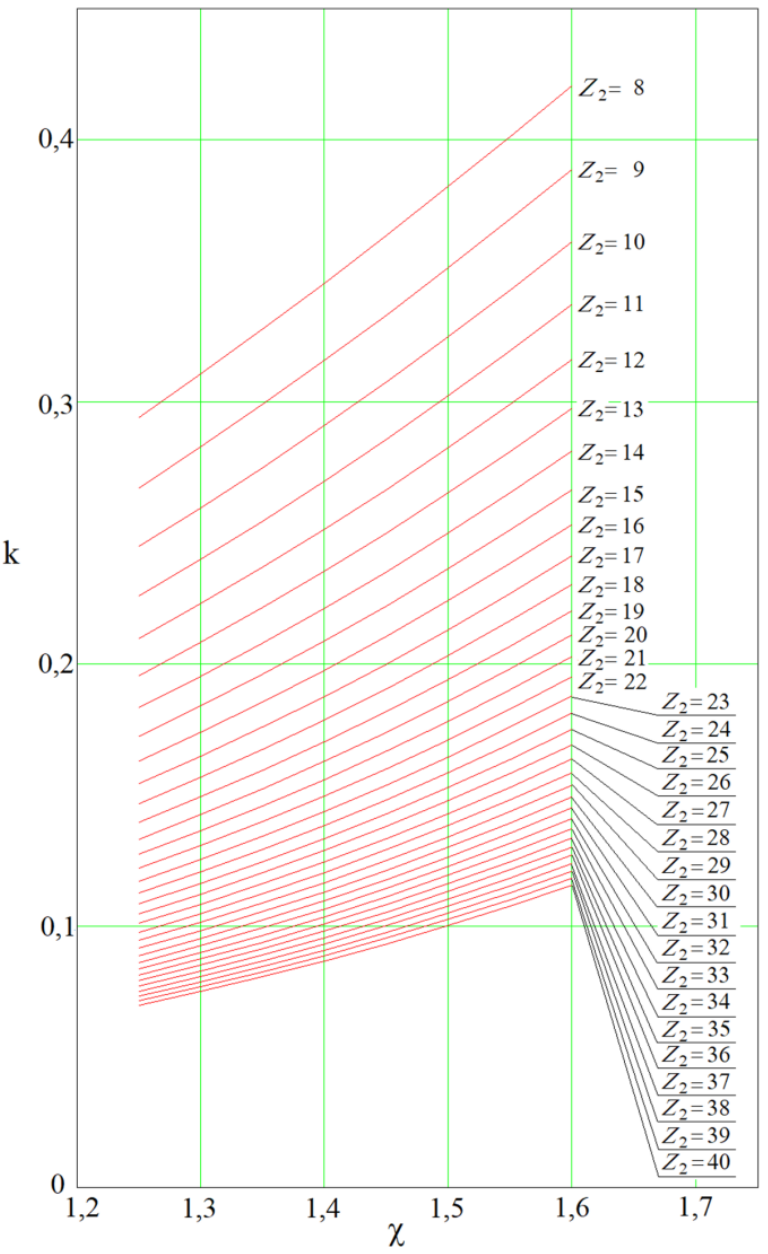

Pис. 3. Гистограмма определения коэффициента $k$ в зависимости от ұ и числа тел качения $Z_{2}$

Fig. 3. Histogram for determination of coefficient $k$ depending on $\chi$ and number of rolling bodies $Z_{2}$

Так, найдя из гистограммы (рис. 3) значение коэффициента $k$, из выражения (12) определяем радиус окружности центров тел качения $r_{c}$ и принимаем окончательное его значение, удовлетворяющее условию контактной прочности. Далее уточняем радиус центроиды $r_{2}$ передачи с ПТКСО на основании полученного радиуса $r_{\text {c }}$ и выбранного коэффициента $\chi$ из следующего выражения:

$$
r_{c}=r_{2} \chi
$$

Уточнив исходные параметры, повторно определяем угол возникновения максимального контактного напряжения в зацеплении из графика (рис. 4). На графике рис. 4 приведена кривая, соответствующая диапазону чисел тел качения, выбираемых для тяжелонагруженных передач с ПТКСО.

Из графика (рис. 4) уточняется угол $\varphi$, пересчитываются коэффициенты и проверяются расчетные напряжения по условию контактной прочности.

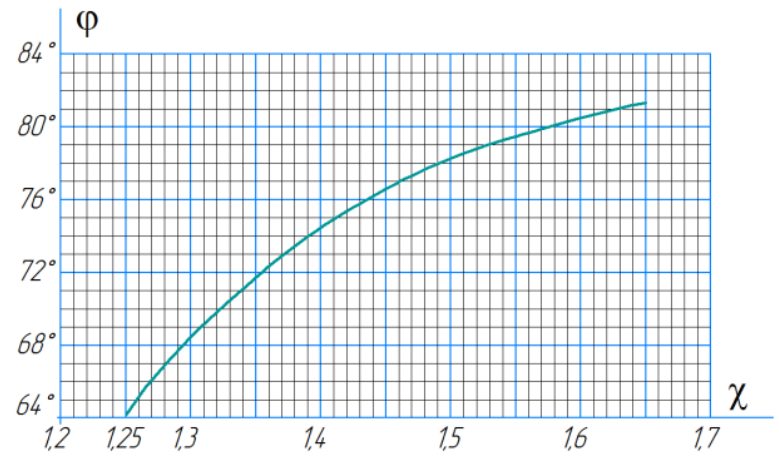

Pис. 4. Изменение угла возникновения максимального контактного напряжения в зависимости от $\chi$

Fig. 4. Change of maximum contact stress occurrence angle depending on $\chi$

Таким образом, определяется приемлемый по условию контактной прочности геометрический параметр передачи с ПТКСО и уточняются исходные параметры циклоидального зацепления, которые можно использовать для дальнейшего проектирования тяжелонагруженных механизмов.

\section{Пример расчета}

Произведем расчет радиуса положения центров тел качения исходя из следующих исходных параметров передачи с ПТКСО: $r_{2}=30 \mathrm{Mм} ; Z_{2}=25 ; \chi=1,4 ; r_{b}=3,5 \mathrm{Mм}$. Пусть крутящий момент на внутреннем колесе $T_{\mathrm{K}}=250 \mathrm{Hм}$ и длина роликов $l_{b}=7 \mathrm{mм}$, а допустимое контактное напряжение для стали ШХ15 $\left[\sigma_{\mathrm{H}}\right]=3000 \mathrm{MПа.}$

По исходным данным для коэффициента $\chi$ и числа тел качения $Z_{2}$ согласно гистограмме (рис. 3) определяем значение коэффициента $k=0,13$. Используя выражения (8), (9) и (12), получим следующее минимально допустимое значение радиуса положения центров тел качения:

$$
r_{c}>0,025 \mathrm{M} .
$$

Зная, что радиус $r_{\mathrm{c}}$ связан с радиусом $r_{2}$ через коэффициент смещения $\chi$ [5], можно уточнить радиус центроиды $r_{2}$, из выражения (13) определяем

$$
\begin{gathered}
r_{c}=r_{2} \chi>0,025, \\
r_{2}>\frac{0,025}{\chi},
\end{gathered}
$$

тогда

$$
r_{2}>0,018 \mathrm{M}
$$

Следовательно, предварительно выбранные исходные параметры, и в частности радиус центроиды $r_{2}$, удовлетворяют условию контактной прочности.

С другой стороны, с учетом уточнения угла $\varphi$ (рис. 4) можно определить максимальный момент, который может обеспечить передача с выбранными параметрами. Так, подставляя в выражение (12) различные значения крутящего момента с шагом 50 Нм, определим, что при $T_{\mathrm{K}}=400$ Нм геометрические параметры $r_{\mathrm{c}}$ и $r_{2}$, полученные из выражений (12) и (13), приблизятся к выбранным предварительно значениям этих параметров. Следовательно, передача, спроектиро- 
ванная по выбранным исходным данным (начало примера), сможет обеспечить передачу крутящего момента до 400 Нм.

\section{Заключение}

Построена гистограмма определения кинематического коэффициента передачи с промежуточными телами качения и свободной обоймой по коэффициенту смещения в зависимости от числа тел качения для предварительного определения геометрических параметров передачи с промежуточными телами качения и свободной обоймой; получено выражение (12), позволяющее определить минимально допустимое значение геометрического параметра $r_{\mathrm{c}}$ для передачи с

\section{СПИСОК ЛИТЕРАТУРЫ}

1. Панкратов Э.Н. Проектирование механических систем автоматизированных комплексов для механообрабатывающего производства. Практикум лидера-проектировщика. - Томск: ТГУ, 1998. - 296 с.

2. Анализ возможности применения редукторного привода в трансмиссии геохода / В.В. Аксенов, А.Б. Ефременков, М.Ю. Блащук, Я.Г. Рыльцева // Вестник науки Сибири. 2012. - № 1 (2). - C. 372-378. URL: http://earchive.tpu.ru/ bitstream/11683/16025/1/279.pdf (дата обращения 07.06.2021).

3. Томилин А.К., Пашков Е.Н., Зиякаев Г.Р. Автобалансировка высокоскоростной двухсекционной газовой центрифуги для обогащения урана // Обогащение руд научно-технический и производственный журнал. - 2019. - № 6. - С. 49-54.

4. Ivkina O.P., Ziyakaev G.R., Pashkov E.N. Mathematic study of the rotor motion with a pendulum selfbalancing device // Journal of Physics: Conference Series - 2016. - № 744. - 8 p.

5. Ефременков Е.А. Разработка методов и средств повышения эффективности передач с промежуточными телами качения: дис. ... канд. техн. наук. - Томск, 2002. - 126 с.

6. Lustenkov M.E. Experimental estimation of efficiency and kinematic accuracy of a spherical roller transmission // IOP Conf. Series: Materials Science and Engineering. - 2020. - № 1118. - 6 p.

7. Prudnikov A.P. Thermal analysis of transmission with intermediate rolling bodies // AER-Advances in Engineering Research (AviaENT 2018). - 2018. - V. 158. - P. 338-342.

8. Lustenkov M.E., Moiseenko A.N. Analysis of contact strength of spherical roller transmission with double-row pinion // IOP Conf. Series: Materials Science and Engineering. - 2021. - № 1118. $6 \mathrm{p}$.

9. An I-Kan, Il'in A.S., Lazurkevich A.V. Load analysis of the planetary gear train with intermediate rollers. Part 2 // IOP Conf Series: Materials Science and Engineering. - 2016. № 124. -6 p.

10. Exposing static indeterminacy of dimensioned gear with packing rolling element / O.P. Ivkina, A. I-Kan, A.V. Cheremnov, E.N. Pashkov $/ / 7^{\text {th }}$ International Forum on Strategic Technology, Conference: Strategic Technology (IFOST2012). - Tomsk, 2012. -6357717 .

11. Lustenkov M.E. Planetary ball transmissions: strength calculations // Russian Engineering Research. - 2010. - V. 30. - № 9. - P. 862-866. промежуточными телами качения и свободной обоймой из условия контактной прочности. По этому параметру в дальнейшем можно скорректировать исходные параметры передачи с учетом прочности. Полученное выражение является основным для расчета на прочность передачи с промежуточными телами качения и свободной обоймой и механизмов на ее основе и может быть использовано в предварительных инженерных расчетах передачи с промежуточными телами качения и свободной обоймой.

Исследования выполнены при поддержке по программе развития Национального исследовательского Томского политехнического университета.

12. Lustenkov M.E., Lustenkova E.S. Load capacity of spherical roller transmission with double-row pinion // IOP Conf. Series: Materials Science and Engineering. -2019 . - № 795. -6 p.

13. Li Xin Xu. A dynamic model to predict the number of pins to transmit load in a cycloidal reducer with assembling clearance // Proc IMechE Part C: J Mechanical Engineering Science. - 2018. V. 233 (12). - P. 4247-4269.

14. Hsieh C.F. Dynamics analysis of cycloidal speed reducers with pinwheel and nonpinwheel designs // Journal of Mechanical Design. - 2014. - V. 136. - № 9. - 11 p.

15. A semi-analytical load distribution model for cycloid drives with tooth profile and longitudinal modifications / Ting Zhang, Xuan Li, Yawen Wang, Lining Sun // Applied Sciences. - 2020. № 10. $-19 \mathrm{p}$.

16. Transmission performance analysis of RV reducers influenced by profile modification and load / H. Wang, Z.Y. Shi, B. Yu, H. Xu // Applied Sciences. - 2019. - № 9. -19 p.

17. Meshing contact analysis of cycloidal-pin gear in RV reducer considering the influence of manufacturing error / T. Li, M. Tian, H. Xu, X. Deng, J. Su // Journal of the Brazilian Society of Mechanical Sciences and Engineering. - 2020. - № 42. - P. 1-14.

18. Леликов О.П. Основы расчета и проектирования деталей и узлов машин. 4-е изд. перераб. и доп. - М.: Машиностроение, 2021. $-464 \mathrm{c}$

19. Яковлев А.Ф., Лобастов В.К. Определение контактной податливости высших пар зацеплений планетарных циклоидальных редукторов и гидромоторов // Вестник машиностроения. 1996. - № 9. - С. 3-7.

20. Тюняев А.В., Звездаков В.П., Вагнер В.А. Детали машин. 2-е изд., испр. и доп. - СПб.: Лань, 2021. - 736 с.

21. Ефременков Е.А. Определение усилий в передаче с промежуточными телами качения и свободной обоймой // Автоматизация и прогрессивные технологии в атомной отрасли: Сборник трудов VI межотраслевой научно-технической конференции. - Новоуральск, 2009. - Т. І. - С. 123-126.

22. Ефременков Е.А., Ан И-Кан Определение радиусов кривизны циклоидальных профилей с использованием метода ЭйлераСавари // Вестник машиностроения. - 2010. - № 10. - С. 47-50.

Поступила 10.11.2021 2.

\section{Информация об авторах}

Eфременков E.A., кандидат технических наук, доцент отделения машиностроения Инженерной школы новых производственных технологий Национального исследовательского Томского политехнического университета; доцент кафедры управления инновациями Томского государственного университета систем управления и радиоэлектроники.

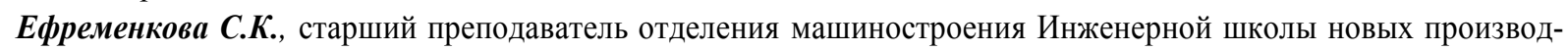
ственных технологий Национального исследовательского Томского политехнического университета. 
UDC 621.831.1:539.4

\title{
DESIGN OF HEAVILY LOADED CYCLOIDAL TRANSMISSION WITH FREE CAGE BASED ON CONTACT STRENGTH
}

\author{
Egor A. Efremenkov1,2, \\ ephrea@mail.ru
}

\author{
Svetlana K. Efremenkova ${ }^{1}$, \\ efremenkova@tpu.ru \\ 1 National Research Tomsk Polytechnic University, \\ 30, Lenin avenue, Tomsk, 634050, Russia. \\ 2 Tomsk State University of Control Systems and Radioelectronics, \\ 40, Lenin avenue, Tomsk, 634050, Russia.
}

\begin{abstract}
The relevance of the research is caused by the need to design heavily loaded mechanisms for mining industry and by the lack of methods for preliminary determination of geometric parameters of cycloidal transmission with intermediate rolling bodies and free cage loading links based on contact strength condition. The technique makes it possible to use compact and energy-saving transmissions with reasonable reliability and durability in drives of mining and transport mechanisms.

The main aim of the research is to identify the dependence of one of the main geometric parameters for transmission with intermediate rolling bodies and a free cage from the contact strength condition, as well as to develop graphic materials for choosing the transmission kinematic coefficient included in the final dependence.

Objects of the research are the cycloidal profiles of the transmission gears at the points of contact with intermediate rolling bodies, as well as the main geometric parameters of the gears necessary for their design.

Methods: analytical methods of the gear engagement theory and stress-strain state of the part.

Results. The authors have given basic equations for determining the geometric parameter of transmission with intermediate rolling bodies and a free cage from the condition of contact strength; an expression was obtained for determining the minimum permissible value of the geometric parameter of transmission with intermediate rolling bodies and a free cage. The paper introduces the histogram for determining the kinematic coefficient of the transmission from a given displacement coefficient and the number of rolling bodies. The results will contribute to the design of reliable, compact, energy-efficient mechanisms for difficult working conditions, which is especially in demand in the mining and oil and gas industries.
\end{abstract}

\section{Key words:}

Heavily loaded mechanism, mining equipment, cycloidal transmission, intermediate rolling bodies, contact strength condition, cycloidal profile, kinematic coefficient, free cage.

The research was supported by the program of development of National Research Tomsk Polytechnic University.

\section{REFERENCES}

1. Pankratov E.N. Proektirovanie mekhanicheskikh sistem avtomatizirovanykh kompleksov dlya mekhanoobrabatyvaushchego proizvodstva [Designing of mechanical systems of the automated complexes for mechano-machining manufacturing. A practical work of the leader-designer]. Tomsk, Tomsk State University Press, 1998. $296 \mathrm{p}$.

2. Aksenov V.V., Efremenkov A.B., Blaschuk M.Yu., Ryltseva Ya.G. Analysis of the possibility of using the reducing drive in the transmission of geohod. Siberian Journal of Science, 2012, no. 1 (2), pp. 372-378. In Rus. URL: http://earchive.tpu.ru/ handle/11683/16025 (accessed 07 June 2021).

3. Tomilin A.K., Pashkov E.N., Ziyakaev G.R. Self-balancing of high-rate two-section gas centrifuge for uranium processing. Obogashchenie Rud, 2019, no. 6, pp. 49-54. In Rus.

4. Ivkina O.P., Ziyakaev G.R., Pashkov E.N. Mathematic study of the rotor motion with a pendulum selfbalancing device. Journal of Physics: Conference Series, 2016, vol. 744, 8 p.

5. Efremenkov E.A. Razrabotka metodov $i$ sredstv povysheniya effektivnosti peredach s promezhutochnymi telami kacheniya. Dis. Kand. nauk [Development of methods and means of increase of effectiveness of transmissions with intermediate rolling bodies. Cand. Diss.]. Tomsk, 2002. 126 p.

6. Lustenkov M.E. Experimental estimation of efficiency and kinematic accuracy of a spherical roller transmission. IOP Conf. Series: Materials Science and Engineering, 2020, vol. 1118, 6 p.
7. Prudnikov A.P. Thermal analysis of transmission with intermediate rolling bodies. AER-Advances in Engineering Research (AviaENT 2018), 2018, vol. 158, pp. 338-342.

8. Lustenkov M.E., Moiseenko A.N. Analysis of contact strength of spherical roller transmission with double-row pinion. IOP Conf. Series: Materials Science and Engineering, 2021, vol. 1118, 6 p.

9. An I-Kan, Il'in A.S., Lazurkevich A.V. Load analysis of the planetary gear train with intermediate rollers. Part 2. IOP Conf. Series: Materials Science and Engineering, 2016, vol. 124, 6 p.

10. Ivkina O.P., I-Kan A., Cheremnov A.V., Pashkov E.N. Exposing static indeterminacy of dimensioned gear with packing rolling element. $7^{\text {th }}$ International Forum on Strategic Technology, Conference: Strategic Technology (IFOST2012). Tomsk, 2012. 6357717.

11. Lustenkov M.E. Planetary ball transmissions: strength calculations. Russian Engineering Research, 2010, vol. 30, no. 9, pp. 862-866.

12. Lustenkov M.E., Lustenkova E.S. Load capacity of spherical roller transmission with double-row pinion. IOP Conf. Series: Materials Science and Engineering, 2019, vol. 795, 6 p.

13. Li Xin Xu. A dynamic model to predict the number of pins to transmit load in a cycloidal reducer with assembling clearance. Proc IMechE Part C: J Mechanical Engineering Science, 2018, vol. 233 (12), pp. 4247-4269.

14. Hsieh C.F. Dynamics analysis of cycloidal speed reducers with pinwheel and nonpinwheel designs. Journal of Mechanical Design, 2014, vol. 136, no. 9, 11 p. 
15. Ting Zhang, Xuan Li, Yawen Wang, Lining Sun. A semi-analytical load distribution model for cycloid drives with tooth profile and longitudinal modifications. Applied Sciences, 2020, vol. 10, 19 p.

16. Wang H., Shi Z.Y., Yu B., Xu H. Transmission performance analysis of RV reducers influenced by profile modification and load. Applied Sciences, 2019, vol. 9, 19 p.

17. Li T., Tian M., Xu H., Deng X., Su J. Meshing contact analysis of cycloidal-pin gear in RV reducer considering the influence of manufacturing error. Journal of the Brazilian Society of Mechanical Sciences and Engineering, 2020, vol. 42, pp. 1-14.

18. Lelikov O.P. Osnovy rascheta $i$ proektirovaniya detaley $i$ uzlov mashin [Basis of calculation and design of machine parts and assemblies]. Moscow, Mashinostroenie Publ., 2021. 464 p.

19. Yakovlev A.F., Lobastov V.K. Opredelenie kontaktnoy podatlivosti vycshikh par zatsepleniy planetarnykh tsykloidalnykh reduktorov i gidromotorov [Determination of higher pairs contact compliance of engagements for planetary cycloid reduces and hydraulic motors]. Vestnik mashinostroeniya, 1996, no. 9, pp. 3-7.
20. Tunyaev A.V., Zvezdakov V.P., Vagner V.A. Detali mashin [Machine partsl]. St-Petersburg, Lan Publ., 2021. 736 p.

21. Efremenkov E.A. Opredelenie usiliy v peredache s promezhutochnymi telami kacheniya i svobodnoy oboymoy [The forces determination in transmission with intermediate rolling bodies and free cage]. VI mezhotraslevaya nauchnotekhnicheskaya konferentsiya. Avtomatizatsiya i progressivnye tekhnologii $v$ atomnoy otrasli [VI intersectoral scientific and technical conference. Automation and progressive technologies in the nuclear industry]. Novouralsk, Novouralsk State Technological Institute Publ., 2009. Vol. I, pp. 123-126.

22. Efremenkov E.A., An I-Kan. Euler-Savari determination of radii of curvature of cycloid profiles. Russian Engineering Research, 2010, vol. 30, no. 10, pp. 1001-1004

Received: 10 November 2021.

\section{Information about the authors}

Egor A. Efremenkov, Cand Sc., associate professor, National Research Tomsk Polytechnic University; associate professor, Tomsk State University of Control Systems and Radioelectronics.

Svetlana K. Efremenkova, senior lecturer, National Research Tomsk Polytechnic University. 